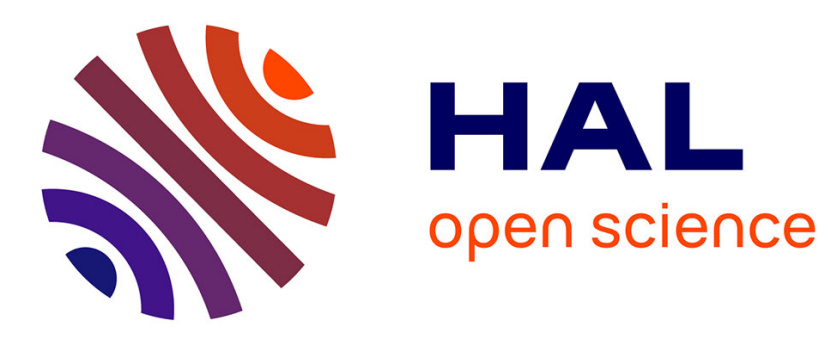

\title{
Undersampled Pulse Width Modulation for Optical Camera Communications
}

Pengfei Luo, Tong Jiang, Paul Anthony Haigh, Zabih Ghassemlooy, Stanislav

\author{
Zvanovec
}

\section{- To cite this version:}

Pengfei Luo, Tong Jiang, Paul Anthony Haigh, Zabih Ghassemlooy, Stanislav Zvanovec. Undersampled Pulse Width Modulation for Optical Camera Communications. 2018 IEEE International Conference on Communications Workshops (ICC Workshops), May 2018, Kansas City, United States. pp.1-6, 10.1109/ICCW.2018.8403732 . hal-02060129

\section{HAL Id: hal-02060129 \\ https://hal-amu.archives-ouvertes.fr/hal-02060129}

Submitted on 7 Mar 2019

HAL is a multi-disciplinary open access archive for the deposit and dissemination of scientific research documents, whether they are published or not. The documents may come from teaching and research institutions in France or abroad, or from public or private research centers.
L'archive ouverte pluridisciplinaire HAL, est destinée au dépôt et à la diffusion de documents scientifiques de niveau recherche, publiés ou non, émanant des établissements d'enseignement et de recherche français ou étrangers, des laboratoires publics ou privés. 


\title{
Undersampled Pulse Width Modulation for Optical Camera Communications
}

\author{
Pengfei Luo ${ }^{1}$, Tong Jiang ${ }^{1}$, Paul Anthony Haigh ${ }^{2}$, Zabih Ghassemlooy ${ }^{3,3 a}$, Stanislav Zvanovec ${ }^{4}$ \\ ${ }^{1}$ Research Department of HiSilicon, Huawei Technologies Co., Ltd, Beijing, China \\ E-mail: \{oliver.luo, toni.jiang\}@hisilicon.com \\ ${ }^{2}$ Department of Electronic and Electrical Engineering, University College London, London, UK \\ Email: p.haigh@ucl.ac.uk \\ ${ }^{3}$ Optical Communications Research Group, NCRLab, Faculty of Engineering and Environment, \\ Northumbria University, Newcastle-upon-Tyne, UK \\ ${ }^{3 a}$ QIEM, Haixi Institutes, Chinese Academy of Sciences, Quanzhou, China \\ Email: z.ghassemlooy@northumbria.ac.uk \\ ${ }^{4}$ Department of Electromagnetic Field, Faculty of Electrical Engineering, \\ Czech Technical University in Prague, 2 Technicka, 16627 Prague, Czech Republic \\ Email:xzvanove@ffel.cvut.cz
}

\begin{abstract}
An undersampled pulse width modulation (UPWM) scheme is proposed to enable users to establish a non-flickering optical camera communications (OCC) link. With UPWM, only a digital light emitting diode (LED) driver is needed to send signals using a higher order modulation. Similar to other undersamplebased modulation schemes for OCC, a dedicated preamble is required to assist the receiver to indicate the phase error introduced during the undersampling process, and to compensate for nonlinear distortion caused by the in-built gamma correction function of the camera. To test the performance of the UPWMbased OCC system, an experimental test-bed is developed. The experimental results show that the proposed system is able to achieve a data rate of 150 bps (spectrum efficiency of $5 \mathrm{bits} / \mathrm{symbol}$ ) at a bit error rate of $6.76 \times 10^{-4}$, which is well below the forward error correction limit of $3.8 \times 10^{-3}$, over a link span of $1 \mathrm{~m}$ using a Huawei Nexus 6P smartphone with a frame rate of 30 fps.
\end{abstract}

Keywords-Optical camera communications, undersampled pulse width modulation, visible light communications

\section{INTRODUCTION}

As superior imaging sensors (ISs) and faster smartphone processors are becoming commonplace, the latest smartphones are as powerful as professional digital single-lens reflex (DSLR) cameras on the photography side. In Dec. 2017, the top 3 ranked mobiles from DxOMark are Google Pixel 2, Apple iPhone X, and Huawei Mate 10 Pro with an overall score of 98, 97, and 97, respectively [1]. According their product specifications, each of these devices are capable of 720p or $1080 p$ video capture at up to 240 frames per second (fps). Such an ability to capture high speed video streams by a smartphone paves the way for future optical camera communication (OCC) technology, mostly for low speed applications such as vehicular communications and indoor positioning [2].

However, most of the cameras embedded in smartphones used today are low frame rate (LFR) based cameras, and the most common frame rates (FRs) are 24, 30, 50 and 60 fps.
According to the Nyquist sampling theorem, if these FRs are adopted for sampling, the transmitted symbol rate $R_{s}$ must be lower than half the sampling rate. However, this will clearly lead to light flickering due to the response time of the human eye. Therefore, a number of techniques have been proposed to support non-flickering OCC using low speed cameras (e.g., $\leq$ $60 \mathrm{fps})$. More precisely, there are three main modulation categories for LFR-based OCC using both global shutter (GS) and rolling shutter (RS) digital cameras: $i$ ) display-based [3], ii) oversampled-based [4, 5], and iii) undersampled-based [6] modulation schemes.

In the display-based OCC system, the data is transmitted via a screen, which displays either observable or unobservable video frames. For example in [3], the useful information is first encoded into 2D visual code, which is then displayed on the screen. The user receives the information by scanning the $2 \mathrm{D}$ visual code using a smartphone's camera and a code scanner application software. Under the oversampled-based OCC system, we have $i$ ) polarization-based modulation, which uses a polarized light generator to emit polarized light with two different polarization states to represent the binary data [4]. Since the change of light polarization state will not be observed, flickering is avoided; and ii) the RS-based OCC system [5], where the signal is transmitted at a very high $R_{s}$ (e.g., several $\mathrm{kBd}$, lower than the RS scanning rate) to eliminate flickering. At the receiver (Rx), a RS-based camera is used to capture the light signal. With the RS receiving mechanism, dark and bright lines, from which the transmitted signal can be extracted, are captured above a normal image frame.

Unlike the oversampled method, in the undersampled-based OCC system, the subcarrier multiplexing scheme is adopted, where the subcarrier frequency higher than the critical flicker frequency (CFF) $f_{\text {cf }}$ of the human eye (i.e., $\sim 100 \mathrm{~Hz}$ [7]) is used to provide constant illumination. Once the FR of the $\mathrm{Rx}$ is equivalent to $R_{s}$ of the transmitter ( $\mathrm{Tx}$ ), a camera is able to undersample the transmitted signal correctly. Many undersampled modulation schemes, along with their dedicated 
date framing structures, have been proposed to establish a nonflickering OCC link as well as to increase the total data throughput.

Table I compares 5 types of undersampled modulation schemes from the perspective of spectral efficiency $\eta_{\text {se }}$ and the driver type. It is clear that for the digital LED driver based modulation schemes: undersampled frequency shift ON-OFF keying (UFSOOK) [8], undersampled phase shift ON-OFF keying (UPSOOK) [9], and undersampled modulation with wavelength division multiplexing (WDM) [10], the spectral efficiencies are lower than $3 \mathrm{bit} /$ frame/LED. Note that, since a three-channel (red, green and blue) based LEDs are employed in the WDM system, for each LED chip the actual $\eta_{\text {se }}$ is still 1 bit/frame/LED. For the analogue LED driver based modulation schemes such as undersampled $m$-ary pulse amplitude modulation (UPAM) $[11,12]$ and undersampled $m$-ary colour shift keying (UCSK) [13] $\eta_{\mathrm{se}}=\log _{2}(m)$ bit/frame/LED. Therefore, for $m>2$, the spectral efficiencies of analogue LED driver based schemes are higher than that of digital LED driver based schemes.

TABLE I. COMPARISON OF UNDERSAMPLED MODULATION SCHEMES

\begin{tabular}{|c|c|c|}
\hline $\begin{array}{c}\text { Name of the modulation } \\
\text { scheme }\end{array}$ & $\begin{array}{c}\text { Spectral efficiency } \eta_{\text {se }} \\
\text { (bits/frame/LED) }\end{array}$ & Driver type \\
\hline UFSOOK & 0.5 & Digital \\
\hline UPSOOK & 1 & Digital \\
\hline $\begin{array}{c}\text { Undersampled modulation } \\
\text { with WDM }\end{array}$ & 3 & Digital \\
\hline$m$-ary UPAM & $\log _{2}(m)$ & Analogue \\
\hline$m$-ary UCSK & $\log _{2}(m)$ & Analogue \\
\hline
\end{tabular}

In this paper, a digital LED driver based OCC modulation scheme named undersampled pulse width modulation (UPWM) is proposed. With UPWM and a signal LED chip, $\eta_{\mathrm{se}}>1$ bit/frame/LED could be easily achieved, thus exceeding those currently available in the literature. An analogue-to-digital converter (ADC), therefore, may not be required, thus leading to a reduced energy consumption and production cost while maintaining higher $\eta_{\text {se }}$, which is advantageous.

The rest of the paper is organized as follows: Section II presents the principle of the proposed UPWM scheme. In Section III, an offline UPWM OCC test-bed is built, and biterror-rate (BER) results with different modulation orders are measured with a real smartphone Nexus 6P. Finally, conclusions and future works are given in Section IV.

\section{PRINCIPLES OF UPWM}

Generally, a camera with a FR and a shutter speed of $f_{\text {cam }}$ and $t_{s h}$, respectively can be considered as an integrate-anddump Rx with a sampling frequency of $f_{\text {cam }}$ (or a sampling duration $\left.t_{c}=1 / f_{\text {cam }}\right)$ and an integration time of $t_{s h}$. More precisely, during the camera exposure process, each pixel captures light for a short period $t_{s h}$, and the electrical signal generated by the pixel is then integrated. Finally, an RGB value of that pixel is generated. In order to ensure that a camera correctly samples all transmitted information with an undersampling mechanism, the Tx sends the information at $R_{\mathrm{s}}$, which is matched to the FR of the Rx.

In this section, five main techniques are presented: pulse width modulation (PWM), UPAM, UPWM, non-linear compensation, and framing structure.

\section{A. $\quad P W M$}

PWM is widely used for simulating an analogue output with digital means, such as provisioning control of an LED's brightness, or the temperature of a heater. Normally, a PWM signal can be described by two parameters: the frequency (or period) and the duty cycle (DC). A linear camera (gamma $=1$, more information about gamma can be found in [14]) with a shutter speed $t_{1}$ can be used to capture an image of an LED driven by a PWM signal with a fixed period $t_{p w m}$ (where $t_{p w m}$ $\left.<<t_{1}\right)$ and a $50 \%$ DC. The result will be recording of $50 \%$ brightness due to the integrate-and-dump process. Therefore, as long as $t_{p w m}$ is much shorter than the camera shutter speed, the LED image with different brightness levels can be captured by simply changing the DC of the PWM signal.

\section{B. UPAM}

As outlined in [11], in UPAM the input binary data stream is first mapped onto the $m$-ary PAM constellation. Next, UPAM is generated by amplitude modulation of a square wave subcarrier signal. Fig. 1 (a) \& (b) illustrate waveforms of the original baseband data $A(n)$ and the corresponding 4-UPAM signal $B(t)$, respectively. In order to ensure that UPAM does not introduce flickering and is correctly sampled by a camera at a frequency of $f_{\text {cam }}$, the subcarrier's frequency $f_{s}$ must be $\geq 200$ $\mathrm{Hz}, t_{s h}$ must be $<1 /\left(2 f_{s}\right)$, and the UPAM symbol rate must equal to $f_{\text {cam }}$.

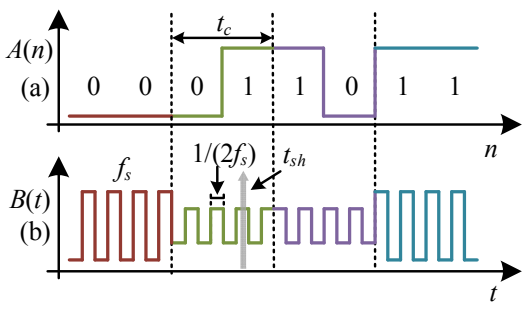

Fig. 1. (a) An example of binary data waveform, and (b) the corresponding 4-ary UPAM waveform with a camera sampling strobe.

\section{UPWM}

Inspired by the UPAM scheme described in the previous subsection, which is a multi-level non-flickering modulation, the UPWM scheme is proposed for the first time in this paper, as a 2-level non-flickering modulation. To maintain the high $\eta_{\text {se }}$ exhibited by UPAM, while decreasing the total cost (i.e., by removing the digital-to-analogue converter), the PWM and the undersampled-based modulation are combined to form the UPWM scheme. Fig. 2 illustrates a block diagram of an OCC system with the UPWM scheme.

1) Tx Side 
At the Tx side, an $m$-ary DC mapper, which maps $A(n)$ to $m$-ary DC sequences $B(m)$. TABLE II. lists a mapping rule, where 2-bit of binary data is mapped into a 4-ary DC sequence $(20 \%, 40 \%, 60 \%, 80 \%)$. Next, $B(m)$ is fed into a UPWM modulator to generate the non-flickering signal. Following the inclusion of a preamble signal $P(t)$, which will be in detailed discussed in Section IIE, the final UPWM signal $C(t)$ is used for intensity modulation of the LED light source via an LED driver module.
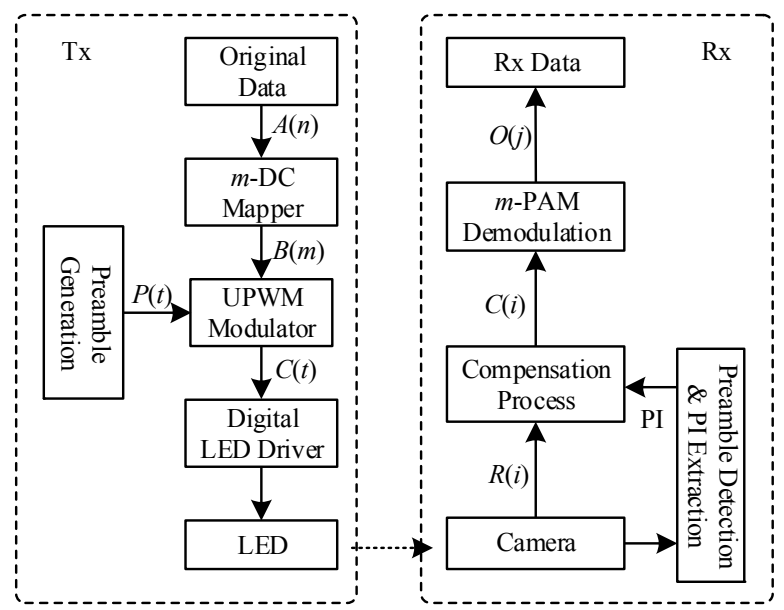

Fig. 2. The block diagram of a UPWM OCC system.

TABLE II. AN EXAMPLE OF A 4-ARY DC MAPPING RULE

\begin{tabular}{|c|c|}
\hline Bits $\boldsymbol{A}(\boldsymbol{n})$ & DC Symbols $\boldsymbol{B}(\boldsymbol{m})$ \\
\hline 00 & $20 \%$ \\
\hline 01 & $40 \%$ \\
\hline 10 & $60 \%$ \\
\hline 11 & $80 \%$ \\
\hline
\end{tabular}

Fig. 3 presents a block diagram of a UPWM modulator. As can be observed if one $B(m)$ symbol is fed into the UPWM modulator, the complementary operation module first generates a DC value of $1-B(m)$, then both DC values $B(m)$ and $1-B(m)$ are used to generate two PWM waveform segments $W_{1}$ and $W_{2}$. Both $W_{1}$ and $W_{2}$ must share the same duration of $t_{c} /(2 k)$, where $k$ enables to ensure flickerless illumination condition $t_{c} /(2 k)<$ $f_{c f}$. The PWM cycle period of both $W_{1}$ and $W_{2}$ is suggested to be $t_{p}$, which might be shorter than $t_{s h} / 5$, to ensure the captured LED brightness level could reflect the DC symbol $B(m)$. Finally, the concatenation and repetition module first generates $k$-segment of both $W_{1}$ and $W_{2}$. This module constantly concatenates all $W_{1}$ and $W_{2}$ segments one after another to form a UPWM symbol $C(t)$ with a duration of $t_{c}$.

To provide an intuitive understanding of the UPWM scheme, Fig. 4 illustrates three waveforms at the Tx side, corresponding to the labels in Fig. 2. From Fig. 4(c), we can see that each UPWM symbol consists of $2 k$ colour patterns (here $k=2$ ), and each colour pattern in the upper part of Fig. 4(c) represents a segment of PWM waveform with a determined duty cycle, a duration of $t_{c} /(2 k)$, and a PWM cycle period of $t_{p}$. Consequently, the generated UPWM signal has an average DC of $50 \%$, and it will not cause flickering. When a camera is used to record video of an LED, which is emitting the UPWM signal, the camera should receive multiple PWM cycles. Therefore the captured brightness level could reflect the DC of the received waveform, however, the captured brightness level may be equal to $B(m)$ or $1-B(m)$, depending on the sampling position. In order to eliminate the uncertainty, a framing structure is proposed which will be in the following subsection.

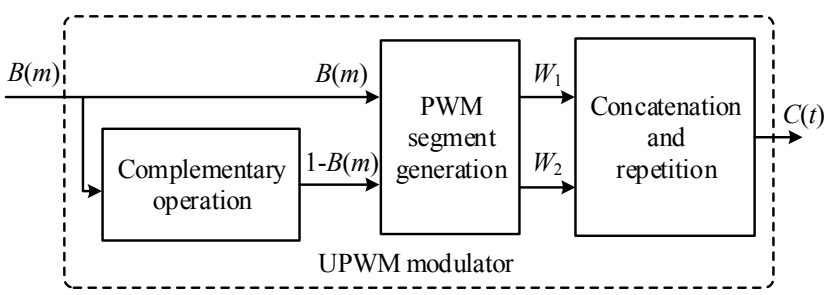

Fig. 3. The block diagram of a UPWM modulator.

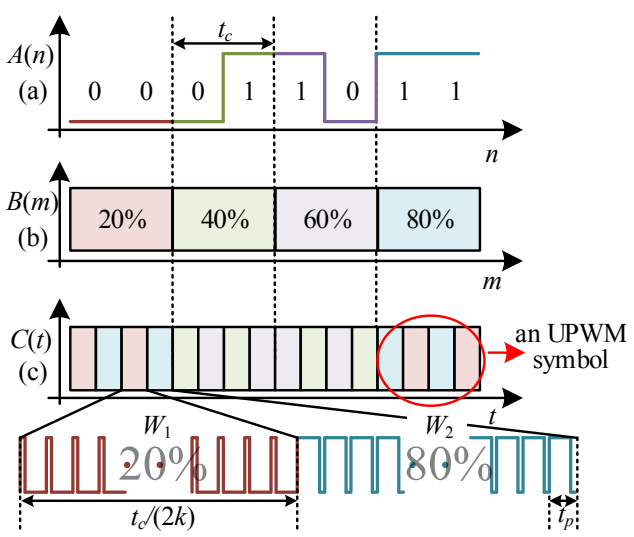

Fig. 4. (a) An example of binary data waveform, (b) the corresponding duty cycle value to generate a UPWM waveform, and (c) the corresponding UPWM waveform.

\section{2) Rx Side}

Following free-space propagation, at the $\mathrm{Rx}$, a camera is employed to detect and record the incoming optical signal. As shown in Fig. 2, the camera outputs are applied to the preamble detection and PI extraction as well as the compensation modules. The output of the compensation module $C(i)$ is passed applied to the $m$-PAM demodulator to regenerated estimated transmitted input data stream $O(j)$. Fig. 5 presents two possible received waveform groups. It is clear that phase error (PE) is likely to occur during the receiving process, since the camera undersamples the incoming signal. Fig. 5(a.II) and (b.II) show the waveforms for the cases without and with the PEs, respectively. 


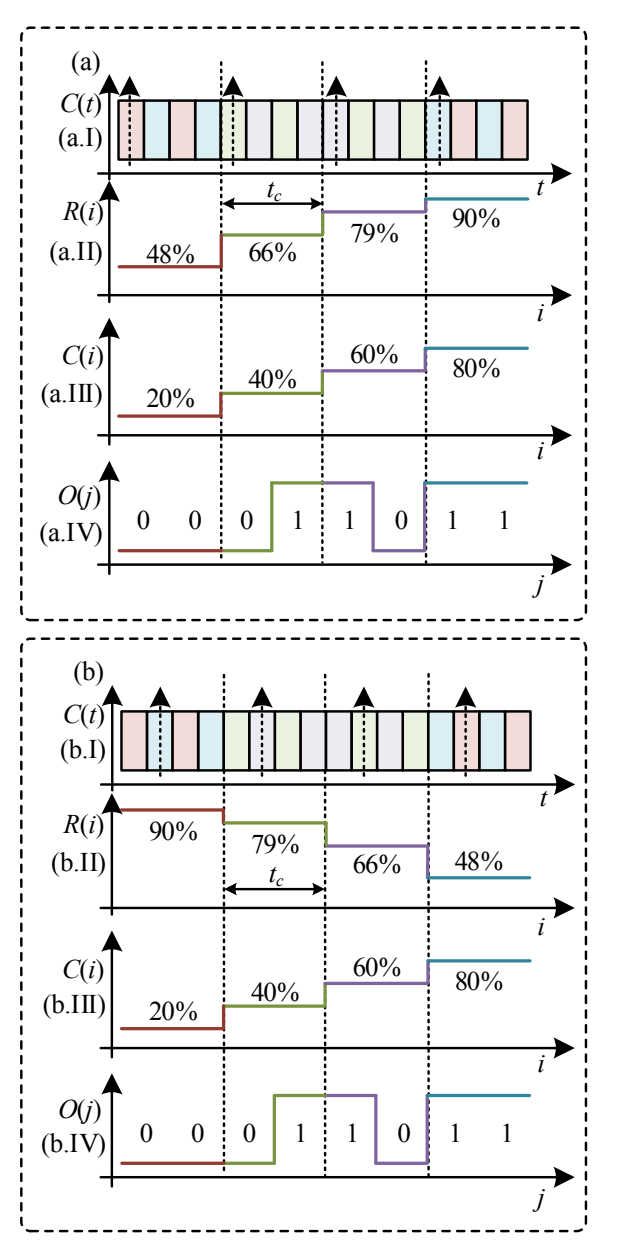

Fig. 5. Two possible received waveform groups: (a) without PE, (b) with PE.

Note that, both PE and nonlinear distortions might be introduced during the undersampling and image digitization processes. However, the Rx still has the ability to compensate for these distortions with the help of preamble detection and preamble information (PI) extraction and compensation modules. More specifically, a preamble $P(t)$ is attached before each group of $m$-ary DC symbols prior to transmission, see Fig. 2 . Therefore, when the preamble is captured by the camera, the PI that assists the compensation process can be extracted. This will be expanded upon in the following subsections.

\section{Nonlinear Compensation}

Gamma correction is normally applied during the digitization process to mimic the nonlinear behaviour of human eyes. Therefore, the original binary data sequence cannot be decoded from the captured LED brightness unless both the sampling phase uncertainty and the nonlinearity-induced problems are resolved. Therefore, a post-compensation (POC) technique [12] is introduced in this paper.

\section{E. Framing Structure}

In this section, a basic framing structure, which consists of a dedicated preamble field and a payload section, is proposed to address both the PE and the nonlinear distortion. The preamble has three main functions: $i$ ) frame synchronization by detecting the unique brightness sequence (e.g., minimum, maximum and ascending or descending sequence); ii) detection and indication of the undersampling induced PE; and iii) nonlinear information acquisition - by obtaining nonlinear distorted sequence. Fig. 6 illustrates the proposed preamble and how it enables the $\mathrm{Rx}$ to decode the sampled brightness. More specifically, the preamble consists of $m+2$ symbols: $i$ ) $0 \%$; ii) all $m$-ary DCs with equidistant intervals denoted by $D_{1} \sim D_{m}$; iii) $100 \%$; and $i v$ ) all DCs of the preamble arranged in ascending order, see Fig. 6(a). Note that, all $m$-ary DCs with equidistant intervals are larger than $0 \%$ and smaller than $100 \%$. Fig. 6(b) depicts the corresponding simplified UPWM waveform. Note, different colours represent the PWM waveform segments with different DCs. Therefore, when no PEs, a changing ascending brightness levels can be received as shown in the upper subfigure of Fig. 6(c). However, if the camera sampling arrows is within the duration of the PWM segments with a complementary DC, a descending brightness levels will be observed as illustrated in Fig. 6(d).

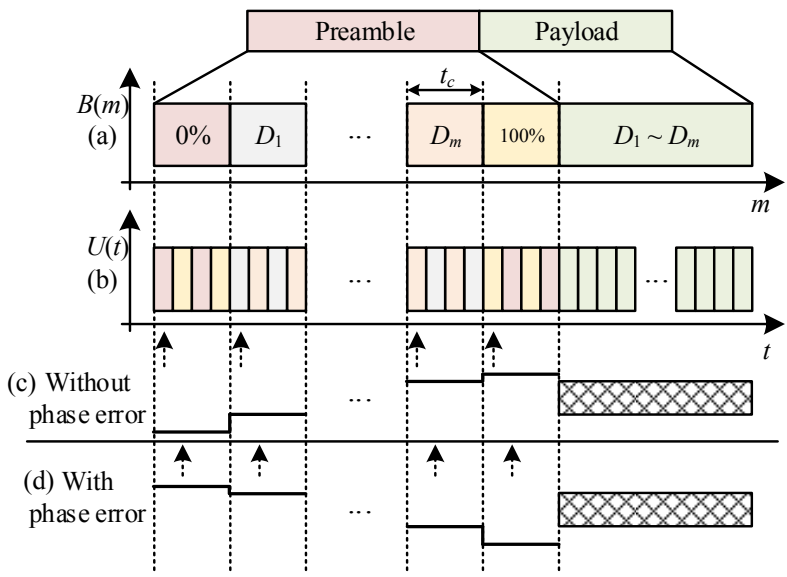

Fig. 6. (a) Preamble + payload data structure, (b) corresponding UPWM signal, and (c) and (d) two undersampled results for the same transmitted signal sampled by two groups of arrows with different phases.

From Fig. 6(c) and (d), we can also see that the ascending or descending brightness levels are no longer equidistantly distributed, and the recorded brightness values of the UPWM symbol with both $0 \%$ and $100 \%$ DCs are either brightest or darkest among all captured brightness values. Thus, if a brightness sequence with an ascending order is received, see Fig. 6(c), then the received sequence is indeed the preamble signal undersampled but without the PE. Thus, the nonlinear curve can be obtained in a straightforward manner from this non-equidistantly distributed sequence using an interpolation method. Similarly, if a brightness sequence with a descending order is received, see Fig. 6(d), then the special sequence is a preamble signal undersampled with the PE, and hence, the nonlinear curve can also be obtained by using an interpolation method. Therefore, with no PE first the payload section must be compensated for nonlinearities and then normalised prior to demodulation using the standard $m$-ary PAM demodulator. However, if the PE is detected using the preamble, then the payload needs to be compensated for nonlinearities first, next 
normalised prior to performing PE compensation. Finally, the signal is demodulated based on the $m$-ary PAM demodulator in the same manner.

\section{EXPERIMENTAL SETUP AND RESULTS}

\section{A. Experimental Setup}

In order to evaluate the performance of the proposed UPWM OCC system, an experimental test-bed was developed with the offline-based data processing, as shown in Fig. 7. All the key system parameters are sown in TABLE III. .

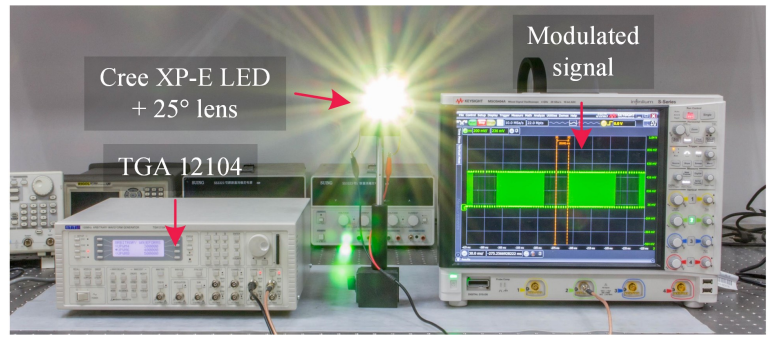

Fig. 7. Experimental setup of the proposed UPWM OCC system.

TABLE III. KEY PARAMETERS OF EXPERIMENT

\begin{tabular}{|c|c|}
\hline Parameter & Value \\
\hline Modulation orders & $2-32$ \\
\hline PWM duration $t_{p}$ & $1 / 15000 \mathrm{~s}$ \\
\hline UPWM symbol duration $t_{c}$ & $1 / 30 \mathrm{~s}$ \\
\hline Communication distance $d$ & $1-3 \mathrm{~m}$ \\
\hline Shutter speed & $1 / 1500 \mathrm{~s}$ \\
\hline Focal length & $4.67 \mathrm{~mm}$ \\
\hline Frame rate & $30 \mathrm{fps}$ \\
\hline Video resolution & $1280 \times 720$ \\
\hline Camera ISO & 60 \\
\hline
\end{tabular}

At the Tx side, a random binary data sequence is first generated in MATLAB, then UPWM symbols are produced according to the rules given in Section IIC. Next, a preamble is prefixed to every 120 UPWM symbols to form a data frame, which are generated using a TTi arbitrary waveform generator (AWG) TGA12104 for intensity modulation of a Cree XP-E LED with a $25^{\circ}$ lens. At the Rx side, a Nexus 6P smartphone (12.3-megapixel Sony Exmor IMX377 sensor with $1.55 \mu \mathrm{m}$ pixels, f/2.0 aperture) with an open camera application [15] is used to record the LED, which continuously transmits the UPWM signal over a transmission span $d$ of 1 to $3 \mathrm{~m}$. Having captured a short video of the Tx, we carry out the following offline processes at the Rx side.

\section{B. Experimental Results}

Fig. 8 shows four measured 64-quadrature amplitude modulation (QAM) constellation diagrams of the received UPWM signal for $d$ of 1.5 and $3 \mathrm{~m}$, without and with nonlinear compensation. Note that, by combining the two adjacent 8-ary DC signals, a single 64-QAM symbol can be formed. Therefore, all 64-QAM constellation points can be obtained from a series of well-designed 128 8-ary DC symbols. From Fig. 8 (a), it is obvious that for $d=1.5 \mathrm{~m}$, the original received signal is nonlinearly distorted (with uneven distances between constellation points), however following nonlinear compensating all constellation points are approximately equidistantly distributed, see Fig. 8 (b). Similarly, for $d=3 \mathrm{~m}$, both constellation diagrams can be seen in Fig. 8 (c) and (d), albeit with decreased signal to noise ratio (SNR) as expected. Note that, in Fig. 8 (d) although the SNR is reduced the constellation diagram is still clear. Given that, the non-linear compensation process is performed at the $\mathrm{Rx}$ side, the noise variance with a larger bias value will be increased with the POC technique. Consequently, the constellation points located in the upper-right corner of Fig. 8 (b) and (d) display higher levels of distortion.

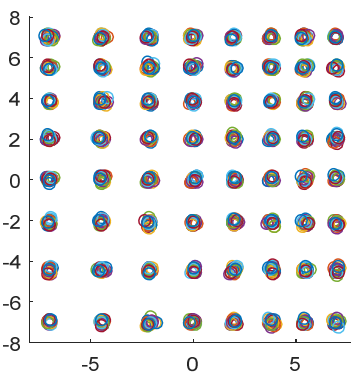

(a)

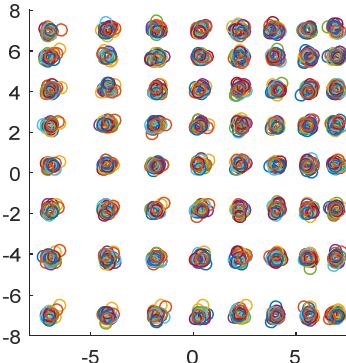

(c)
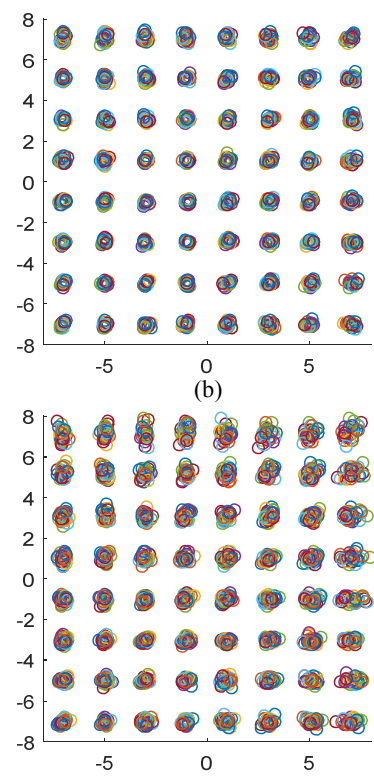

(d)
Fig. 8. Constellation diagrams of 64-QAM signals combined from an 8UPWM signal: (a) the original received signal for $d=1.5 \mathrm{~m}$, (b) the compensated signal for $d=1.5 \mathrm{~m}$, (c) the original received signal for $d=$ $3 \mathrm{~m}$, and (d) the compensated signal for $d=3 \mathrm{~m}$.

In order to assess the feasibility of the proposed scheme, we measure the BER performances for different modulation orders are measured at $d=1 \mathrm{~m}$. For each modulation order, a 10minute video was recorded, then all video files were processed offline in MATLAB. Since the mobile phone introduces a real multi-task device, which runs multiple applications in the background, when the mobile phone was used in a camera receiver mode, it can sometimes drop video frames due to multi-tasking. Although this may not cause a serious problem for entertainment purpose, it could lead to the signal loss in data communications.

In our experiment, both the values of the lost data frame and the BER are measured and are listed in TABLE IV. During the offline process, for any video frame being dropped the affected data frame is then considered as a lost data frame. 
According to the measured data, $0 \sim 10$ dropped data frames are considered lost during about 10 minutes of signal reception. The lost data frames are then removed in order to determine the BER values caused only by the noise and non-ideal non-linear compensation. It is clear that, for all modulation orders the BER values are well below the forward error correction (FEC) limit of $3.8 \times 10^{-3}$ [16], which proves the feasibility of the proposed scheme. In order to reduce the impact of dropped video frames, the data frames with a short payload are proposed. However, a short payload means reduced throughput efficiency.

TABLE IV. MEASURED BER VALUES AND THE TOTAL NUMBER OF RECEIVED SYMBOLS WITH DIFFERENT MODULATION ORDERS

\begin{tabular}{|c|c|c|c|}
\hline $\begin{array}{c}\text { Modulation } \\
\text { order }\end{array}$ & $\begin{array}{c}\text { Reviewed } \\
\text { symbol }\end{array}$ & $\begin{array}{c}\text { Frame number } \\
\text { with lost signal }\end{array}$ & BER \\
\hline 2-PAM & 19609 & 9 & 0 \\
\hline 4-PAM & 22119 & 10 & 0 \\
\hline 8-PAM & 18960 & 0 & 0 \\
\hline 16-PAM & 20956 & 0 & $9.99 \times 10^{-5}$ \\
\hline 32-PAM & 21552 & 6 & $6.76 \times 10^{-4}$ \\
\hline
\end{tabular}

\section{CONCLUSIONS AND FUTURE WORKS}

In this paper, a low cost UPWM scheme was proposed for the low-frame-rate based OCC system. Unlike most other high spectral efficiency undersampled modulation schemes, which require an analogue LED driver to transmit signals, this modulation scheme only requires a digital LED driver to send a non-flickering signal. To address the unsynchronized phase and non-linear distortion induced problems, a unique preamble was proposed and designed. An offline mobile phone-based OCC test-bed was also developed to assess the feasibility of the proposed scheme. The BER values and the dropped video frame numbers for different modulation orders were consequently measured. We showed that for all modulation orders the BER values are well below the FEC limit of $3.8 \times 10^{-3}$, which illustrates the feasibility of the proposed scheme.

Future work on this topic will consider following tasks $i$ ) how to use modulation orders that do not equal to $2^{k}$, where $k$ is an integer; $i$ ) how to combine the undersampled schemes with the rolling shutter based schemes; and iii) how to reduce the impact of dropped video frame on communication performance.

\section{ACKNOWLEDGEMENT}

This work was supported in part by the EPSRC research grant EP/P006280/1: MARVEL and under the Marie Slodowska-Curie ITN grant no. 764461 VisIoN. The Authors would like to acknowledge Professor Min Zhang of Beijing University of Post and Telecommunications for providing experimental resources. P. Luo would like to acknowledge the support from Helen Ouyang.

\section{REFERENCES}

[1] DxOMark. (2018). Mobile reviews. Available: https://www.dxomark.com/category/mobile-reviews/

[2] Z. Ghassemlooy, P. Luo, and S. Zvanovec, "Optical Camera Communications," in Optical Wireless Communications: Springer, 2016 , pp. 547-568.

[3] R. Boubezari, H. L. Minh, Z. Ghassemlooy, and A. Bouridane, "Smartphone Camera Based Visible Light Communication," Journal of Lightwave Technology, vol. 34, no. 17, pp. 4121-4127, 2016.

[4] Z. Yang, Z. Wang, J. Zhang, C. Huang, and Q. Zhang, "Wearables can afford: Light-weight indoor positioning with visible light," in Proceedings of the 13th Annual International Conference on Mobile Systems, Applications, and Services, 2015, pp. 317-330: ACM.

[5] C. Danakis, M. Afgani, G. Povey, I. Underwood, and H. Haas, "Using a CMOS camera sensor for visible light communication," in GC Wkshps, IEEE, 2012, pp. 1244-1248.

[6] P. Luo, M. Zhang, Z. Ghassemlooy, S. Zvanovec, S. Feng, and P. Zhang, "Undersampled-based modulation schemes for optical camera communications," IEEE Communications Magazine, vol. 55, no. 2, 2018

[7] C. S. Herrmann, "Human EEG responses to 1-100 Hz flicker: resonance phenomena in visual cortex and their potential correlation to cognitive phenomena,", Exp Brain Res, vol. 137, no. 3-4, pp. 346-53, Apr 2001.

[8] R. D. Roberts, "Undersampled frequency shift ON-OFF keying (UFSOOK) for camera communications (CamCom)," in WOCC, 2013, pp. 645-648: IEEE.

[9] P. Luo, Z. Ghassemlooy, H. L. Minh, X. Tang, and H.-M. Tsai, "Undersampled phase shift ON-OFF keying for camera communication," in WCSP, 2014, pp. 1-6.

[10] P. Luo, M. Zhang, Z. Ghassemlooy, H. Le Minh, H.-M. Tsai et al., "Experimental Demonstration of RGB LED-Based Optical Camera Communications," IEEE Photonics Journal, vol. 7, no. 5, pp. 1-12, Oct 2015.

[11] P. Luo, Z. Ghassemlooy, H. L. Minh, H.-M. Tsai, and X. Tang, "Undersampled-PAM with subcarrier modulation for camera communications," in $O E C C$, Shanghai, China, 2015, pp. 1-3.

[12] P. Luo, M. Zhang, Z. Ghassemlooy, H. L. Minh, H. M. Tsai et al., "Experimental Demonstration of a 1024-QAM Optical Camera Communication System," IEEE Photonics Technology Letters, vol. 28 no. 2, pp. 139-142, 2016.

[13] P. Luo, Z. Ghassemlooy, S. Zvanovec, S. Feng, P. Zhang, and M. Zhang, "Experimental demonstration of undersampled color-shift keying optical camera communications," in ICCC Workshops, 2017, pp. 1-6.

[14] S. Wright, "Gamma," in Digital Compositing for Film and Video (Third Edition), S. Wright, Ed. Boston: Focal Press, 2010, pp. 305-317.

[15] Open Camera. (2018). Available: https://opencamera.sourceforge.io/

[16] ITU-T, "G.975.1, Forward error correction for high bit-rate DWDM submarine systems," ed: Feb., 2004, p. Appendix I.9. 